\title{
The spectrum of the multiplication operator as- sociated with a family of operators in a Banach space
}

\author{
R. Denk, M. Möller and C. Tretter
}

\begin{abstract}
An operator family of densely defined closed linear operators and the multiplication operator associated with it are considered. The spectrum of this multiplication operator is expressed in terms of the spectra of the operators in the given family.
\end{abstract}

Mathematics Subject Classification (2000). Primary 47A10; Secondary 47A56, 47B40, 47E05, 76B99.

Keywords. Multiplication operator, operator family, vector measure, spectrum.

\section{Introduction}

When considering problems from mathematical physics modelled by linear differential operators, separation of variables (often with respect to the time variable on the one hand and the space variables on the other hand) leads to spectral problems where the spectrum gives information about stability and discrete states. However, a further separation of variables in the space variables is often useful; for example, if differentiation does not occur with respect to all space variables. Therefore, the original spectral problem is split into a family of spectral problems. Here we investigate the question how the spectrum of the original problem can be descibed by the spectra of the operators in this family. This allows, for example, to describe the spectrum of certain PDE problems in terms of spectra of a family of associated ODE problems.

More precisely, we consider an operator family $(A(\rho))_{\rho \in X}$ of closed densely defined operators on a Banach space $E$, where $X$ is a locally compact space. With

The second author has been partially supported through a grant by the NRF of South Africa, GUN 2053746, and by the John Knopfmacher Centre for Applicable Analysis and Number Theory at the University of the Witwatersrand, Johannesburg. 
this family we associate an operator $\mathfrak{A}$ on $L^{p}(X, \mu, E), 1 \leq p<\infty$, for a given Radon measure $\mu$ on $X$ such that

$$
(\mathfrak{A} f)(\rho)=A(\rho) f(\rho), \quad \rho \in X,
$$

which we call a multiplication operator. Our main result describes the spectrum of $\mathfrak{A}$ in terms of the spectra of the operators $A(\rho)$.

There are two main assumptions on the operator family $A(\rho)$, namely that the domains $\mathcal{D}(A(\rho))$ are independent of $\rho$ and that the operator family depends continuously on $\rho$ on a compactification of $X$, where the common domain is equipped with a graph norm. For self-adjoint operators on Hilbert spaces, this concept was developed e.g. in Reed and Simon, [9, Section XIII.16]. There it is only assumed that $A(\rho)$ depends measurably on $\rho$; however, the characterization of the spectrum is more complicated, see [9, Theorem XIII.85]. For not necessarily self-adjoint operators in Hilbert space one can use the theory of direct integrals, see e. g. Azoff, [1], and Dixmier, [5, Chapter II, §2]. For usual multiplication operators, i.e. multiplication by matrix functions, the spectrum has been investigated e. g. by Hardt and Wagenführer in [7].

As was pointed out in [1], in general there is little resemblance between the spectra of the family $A(\rho)$ and the spectrum of $\mathfrak{A}$. Therefore we will require continuity of the family $A(\rho)$; see below for a precise definition. Although our assumptions on $A(\rho)$ seem quite restrictive, many problems in mathematical physics lead to operator functions of this type. We note that a particular example has been studied by Binding and Volkmer in [2] in the setting of two-parameter problems.

In [3] we have considered a particular example from magnetohydrodynamics in $L^{2}$. In this paper we give a more general theoretical background and extend the example into a more general setting. In a forthcoming paper we will consider the more general case that the assumptions on $A(\rho)$ are replaced by the assumption that $A(\rho)$ depends continuously on $\rho$ with respect to the gap topology on the space of closed operators in $H$. This allows the domains of $A(\rho)$ to depend on $\rho$.

The paper is organized as follows. In Section 2 we define the multiplication operator $\mathfrak{A}$ associated with $(A(\rho))_{\rho \in X}$ and prove that $\mathfrak{A}$ is closed. In Section 3 it is shown that the spectrum of $\mathfrak{A}$ is the union of the spectra of $A(\rho)$ over $\rho$ in the compactification of $X$. Results on the point spectrum and the essential spectrum are established in Section 4. In Section 5 results are obtained for cases where $A(\rho)$ is only continuous on $X$. In Section 6 the general results are applied to some classes of examples to illustrate the reduction process.

\section{The multiplication operator associated with an operator family}

Throughout this paper, $X$ denotes a nonempty locally compact Hausdorff space, $\mu$ a Radon measure on $X$ with $\operatorname{supp} \mu=X, E$ a Banach space with norm $\|\cdot\|$, $1 \leq p<\infty$, and

$$
\mathfrak{H}:=L^{p}(X, \mu, E)
$$


the space of $L^{p}$-functions on $X$ with respect to $\mu$ with values in $E$. It is well known that $\mathfrak{H}$ is a Banach space with dual $\mathfrak{H}^{*} \supset L^{p^{\prime}}\left(X, \mu, E^{*}\right)$, see e. g. [6, Theorem III.6.6] and $\left[4\right.$, p. 97], where $1 / p+1 / p^{\prime}=1$. Note that [4] only deals with finite measure spaces, but it is easily seen that finiteness is not needed here. Let $\mathcal{C}(E)$ denote the set of closed operators on $E$. For a subspace $D \subset E$, we denote by $\mathcal{C}_{D}(E)$ the subset of $\mathcal{C}(E)$ consisting of those closed operators $T$ with domain $\mathcal{D}(T)$ being exactly $D$.

On $\mathcal{C}_{D}(E)$ we define a topology as follows. For an operator $G \in \mathcal{C}_{D}(E)$, we denote by $\|\cdot\|_{G}$ the graph norm of $G$ on $D$ given by

$$
\|x\|_{G}:=\left(\|x\|^{p}+\|G x\|^{p}\right)^{\frac{1}{p}}, \quad x \in D,
$$

and set

$$
\rho_{G}(S, T):=\|S-T\|_{G}:=\sup _{\substack{x \in D \\\|x\|_{G}=1}}\|(S-T) x\|, \quad S, T \in \mathcal{C}_{D}(E) .
$$

Then $\rho_{G}$ is a metric on $\mathcal{C}_{D}(E)$. We note that the topology induced by the metric $\rho_{G}$ does not depend on the choice of the operator $G$ since all the graph norms on $D$ are equivalent by the closed graph theorem, and that $\left(\mathcal{C}_{D}(E),\|\cdot\|_{G}\right)$ can be identified with a subset of the space $\mathcal{B}\left(\left(D,\|\cdot\|_{G}\right), E\right)$ of bounded linear operators from $\left(D,\|\cdot\|_{G}\right)$ to $E$.

Let $Y$ be a compactification of $X$. We consider an operator function

$$
A: Y \rightarrow \mathcal{C}(E)
$$

with the following properties:

(a) $D=\mathcal{D}(A(\rho)), \rho \in Y$, is independent of $\rho$ and a dense subspace of $E$,

(b) $A: Y \rightarrow \mathcal{C}_{D}(E)$ is continuous,

where $\mathcal{C}_{D}(E)$ is equipped with the above mentioned topology.

Proposition 2.1. Let assumptions (a) and (b) be satisfied. Then there are positive constants $c_{G}, m_{G}$ such that

$$
\|A(\rho)\|_{G} \leq c_{G}, \quad \rho \in Y
$$

and

where $M_{G}:=\left(1+c_{G}^{p}\right)^{\frac{1}{p}}$.

$$
m_{G}\|x\|_{G} \leq\|x\|_{A(\rho)} \leq M_{G}\|x\|_{G}, \quad x \in D, \rho \in Y,
$$

Proof. The existence of $c_{G}$ such that (2.1) holds is an immediate consequence of assumption (b) and the compactness of $Y$.

Now let $x \in D$ and $\rho \in Y$. Then

$$
\|x\|_{A(\rho)}^{p}=\|x\|^{p}+\|A(\rho) x\|^{p} \leq\|x\|^{p}+c_{G}^{p}\left(\|x\|^{p}+\|G x\|^{p}\right) \leq\left(1+c_{G}^{p}\right)\|x\|_{G}^{p},
$$

which proves the right inequality in $(2.2)$.

Assume that the left inequality in (2.2) is false for any positive constant $m_{G}$. Then there are a sequence $\left(x_{n}\right)_{1}^{\infty}$ in $D$ and a sequence $\left(\rho_{n}\right)_{1}^{\infty}$ in $Y$ such that $\left\|x_{n}\right\|_{G}=1$ for all $n \in \mathbb{N}$ and $\left\|x_{n}\right\|_{A\left(\rho_{n}\right)} \rightarrow 0$ as $n \rightarrow \infty$. Since $Y$ is compact, there 
is a limit point $\rho \in Y$ of $\left(\rho_{n}\right)_{1}^{\infty}$. The continuity assumption (b) implies that for every $\varepsilon>0$ there is a positive integer $n_{\varepsilon}$ such that $\left\|A\left(\rho_{n_{\varepsilon}}\right)-A(\rho)\right\|_{G}<\varepsilon$ and $\left\|x_{n_{\varepsilon}}\right\|_{A\left(\rho_{n_{\varepsilon}}\right)}<\varepsilon$. This leads to

$$
\begin{aligned}
\left\|x_{n_{\varepsilon}}\right\|_{A(\rho)}^{p} & =\left\|x_{n_{\varepsilon}}\right\|^{p}+\left\|A(\rho) x_{n_{\varepsilon}}\right\|^{p} \\
& \leq\left\|x_{n_{\varepsilon}}\right\|^{p}+2^{p-1}\left\|A(\rho) x_{n_{\varepsilon}}-A\left(\rho_{n_{\varepsilon}}\right) x_{n_{\varepsilon}}\right\|^{p}+2^{p-1}\left\|A\left(\rho_{n_{\varepsilon}}\right) x_{n_{\varepsilon}}\right\|^{p} \\
& \leq\left\|x_{n_{\varepsilon}}\right\|^{p}+2^{p-1}\left\|A(\rho)-A\left(\rho_{n_{\varepsilon}}\right)\right\|_{G}^{p}\left\|x_{n_{\varepsilon}}\right\|_{G}^{p}+2^{p-1}\left\|A\left(\rho_{n_{\varepsilon}}\right) x_{n_{\varepsilon}}\right\|^{p} \\
& \leq 2^{p} \varepsilon^{p} .
\end{aligned}
$$

But this contradicts the equivalence of the two graph norms $\|\cdot\|_{A(\rho)}$ and $\|\cdot\|_{G}$.

Proposition 2.2. Let assumptions (a) and (b) be satisfied. Then there is a unique bounded linear operator $\widetilde{A}$ from $L^{p}(X, \mu, D)$ into $L^{p}(X, \mu, E)$ such that $(\widetilde{A} f)(\rho)=$ $A(\rho) f(\rho)$ for all $f \in L^{p}(X, \mu, D)$ and almost all $\rho \in X$.

Proof. Let $f \in L^{p}(X, \mu, D)$ be a simple function, that is, $f=\sum_{i=1}^{n} \chi_{A_{i}} f_{i}$ with measurable $A_{i} \subset X$ and $f_{i} \in D, i=1,2, \ldots, n$, where $\chi_{A_{i}}$ denotes the characteristic function of $A_{i}$. Then

$$
A(\rho) f(\rho)=\sum_{i=1}^{n} \chi_{A_{i}}(\rho) A(\rho) f_{i}
$$

and hence $\rho \mapsto A(\rho) f(\rho)$ is measurable by assumption (b). Further, by (2.1),

$$
\int_{X}\|A(\rho) f(\rho)\|^{p} d \mu(\rho) \leq \int_{X}\|A(\rho)\|_{G}^{p}\|f(\rho)\|_{G}^{p} d \mu(\rho) \leq c_{G}^{p} \int_{X}\|f(\rho)\|_{G}^{p} d \mu(\rho) .
$$

Hence there is a unique bounded linear operator defined on the subset of simple functions of $L^{p}(X, \mu, D)$ with the desired property. Since this subset is dense in $L^{p}(X, \mu, D)$, see [6, Corollary III.3.8], the proof is complete.

Theorem 2.3. Let assumptions (a) and (b) be satisfied. Then the operator $\mathfrak{A}$ in $\mathfrak{H}=L^{p}(X, \mu, E)$ defined on $\mathcal{D}(\mathfrak{A}):=L^{p}(X, \mu, D)$ by $\mathfrak{A} f=\widetilde{A} f$, i.e.

$$
(\mathfrak{A} f)(\rho)=A(\rho) f(\rho)
$$

for $f \in L^{p}(X, \mu, D)$ and almost all $\rho \in X$, is closed.

Proof. Let $\left(\mathfrak{f}_{n}\right)_{1}^{\infty}$ be a sequence in $L^{p}(X, \mu, D)$ such that $\mathfrak{f}_{n} \rightarrow \mathfrak{f}$ and $\mathfrak{A} \mathfrak{f}_{n} \rightarrow \mathfrak{g}$ in $L^{p}(X, \mu, E)$ for some $\mathfrak{f}, \mathfrak{g} \in L^{p}(X, \mu, E)$. As every $L^{p}$-convergent sequence contains a subsequence converging almost everywhere, see [6, Theorem III.3.6 and Corollary III.6.13], we may assume that $\mathfrak{f}_{n}(\rho) \rightarrow \mathfrak{f}(\rho),\left(\mathfrak{A f}_{n}\right)(\rho) \rightarrow \mathfrak{g}(\rho)$ for almost all $\rho \in X$. Hence, since $\left(\mathfrak{A f}_{n}\right)(\rho)=A(\rho) \mathfrak{f}_{n}(\rho)$ by definition of $\mathfrak{A}$ and since $A(\rho)$ is closed, it follows that $\mathfrak{f}(\rho) \in D$ and $A(\rho) \mathfrak{f}(\rho)=\mathfrak{g}(\rho)$ for almost all $\rho \in X$.

To finish the proof we have to show that $\mathfrak{f} \in L^{p}(X, \mu, D)$, for which it suffices to prove that $\left(\mathfrak{f}_{n}\right)_{1}^{\infty}$ is a Cauchy sequence there. In view of Proposition 2.1 we 
conclude that

$$
\begin{aligned}
\left\|\mathfrak{f}_{n}-\mathfrak{f}_{m}\right\|_{L^{p}(X, \mu, D)}^{p} & =\int_{X}\left\|\mathfrak{f}_{n}(\rho)-\mathfrak{f}_{m}(\rho)\right\|_{G}^{p} d \mu(\rho) \\
& \leq m_{G}^{-p} \int_{X}\left\|\mathfrak{f}_{n}(\rho)-\mathfrak{f}_{m}(\rho)\right\|_{A(\rho)}^{p} d \mu(\rho) \\
& =m_{G}^{-p} \int_{X}\left(\left\|\mathfrak{f}_{n}(\rho)-\mathfrak{f}_{m}(\rho)\right\|^{p}+\left\|A(\rho)\left(\mathfrak{f}_{n}(\rho)-\mathfrak{f}_{m}(\rho)\right)\right\|^{p}\right) d \mu(\rho) \\
& =m_{G}^{-p}\left[\left\|\mathfrak{f}_{n}-\mathfrak{f}_{m}\right\|_{\mathfrak{H}}^{p}+\left\|\mathfrak{A f}_{n}-\mathfrak{A f}_{m}\right\|_{\mathfrak{H}}^{p}\right] \rightarrow 0
\end{aligned}
$$

as $n, m$ tend to $\infty$, which completes the proof.

Because of (2.3) we call $\mathfrak{A}$ the multiplication operator associated with the operator family $A$. The simplest examples of multiplication operators associated with a family of operators are operators of multiplication by scalar functions or, more generally, by matrix functions. But multiplication operators may also arise from differential operators the highest derivatives of which do not contain derivatives in all directions, see Section 6.

\section{The spectrum of the multiplication operator}

In the following for an operator $T$ we denote its spectrum by $\sigma(T)$, its point spectrum, i. e., the set of its eigenvalues, by $\sigma_{p}(T)$, and its essential spectrum, i.e., the set of all points $\lambda \in \sigma(T)$ where $T-\lambda$ is not a Fredholm operator, by $\sigma_{\text {ess }}(T)$.

Theorem 3.1. Let assumptions (a) and (b) be satisfied. Then the spectra of the operator $\mathfrak{A}$ and the operator family $A$ are related as follows:

$$
\sigma(\mathfrak{A})=\bigcup_{\rho \in Y} \sigma(A(\rho))
$$

Proof. Let $\lambda \in \bigcup_{\rho \in Y} \sigma(A(\rho))$. Choose $\rho_{0} \in Y$ such that $\lambda \in \sigma\left(A\left(\rho_{0}\right)\right)$. In view of assumption (b) we can choose a sequence of open neighbourhoods $Y_{n}$ of $\rho_{0}$ in $Y$ such that $\left\|A(\rho)-A\left(\rho_{0}\right)\right\|_{G}<\frac{1}{n}$ for all $\rho \in Y_{n}$. For $\lambda \in \sigma\left(A\left(\rho_{0}\right)\right)$ there are two cases: either $A\left(\rho_{0}\right)-\lambda$ has a closed range which is a proper subspace of $E$, or there is a sequence $\left(f_{n}\right)_{1}^{\infty}$ in $D$ with $\left\|f_{n}\right\|=1$ and $\left\|\left(A\left(\rho_{0}\right)-\lambda\right) f_{n}\right\|<\frac{1}{n}$. We first consider the second case. Since $X$ is dense in $Y, X_{n}:=Y_{n} \cap X$ is a nonempty open subset of $X$. Since $\mu$ is a Radon measure on the locally compact space $X$ with support $X$, we can find a measurable subset $M_{n}$ of $X_{n}$ such that $\mu\left(M_{n}\right)$ is a finite positive number. Let $\alpha_{n}:=\left(\mu\left(M_{n}\right)\right)^{-\frac{1}{p}}$ and set

$$
\mathfrak{f}_{n}(\rho):=\alpha_{n} \chi_{M_{n}}(\rho) \frac{f_{n}}{\left\|f_{n}\right\|_{G}}, \quad \rho \in X .
$$

Obviously, $\mathfrak{f}_{n} \in L^{p}(X, \mu, D)$ and $\left\|\mathfrak{f}_{n}\right\|_{L^{p}(X, \mu, D)}=1$. Furthermore, 


$$
\begin{aligned}
\left\|(\mathfrak{A}-\lambda) \mathfrak{f}_{n}\right\|_{\mathfrak{H}}^{p} & =\alpha_{n}^{p} \int_{M_{n}}\left\|(A(\rho)-\lambda) \frac{f_{n}}{\left\|f_{n}\right\|_{G}}\right\|^{p} d \mu(\rho) \\
& \leq \alpha_{n}^{p} \mu\left(M_{n}\right) \sup _{\rho \in M_{n}}\left(\left\|A(\rho)-A\left(\rho_{0}\right)\right\|_{G}+\frac{1}{\left\|f_{n}\right\|_{G}}\left\|\left(A\left(\rho_{0}\right)-\lambda\right) f_{n}\right\|\right)^{p} \\
& <\frac{2^{p}}{n^{p}}
\end{aligned}
$$

since $\left\|f_{n}\right\|_{G} \geq\left\|f_{n}\right\|=1$, which proves $\lambda \in \sigma(\mathfrak{A})$.

Now assume that $A\left(\rho_{0}\right)-\lambda$ has a closed range which is a proper subspace of $E$. Then there is $h \in E^{*} \backslash\{0\}$ such that $\left\langle\left(A\left(\rho_{0}\right)-\lambda\right) f, h\right\rangle=0$ for all $f \in D$. Suppose that $\lambda \in \rho(\mathfrak{A})$. Choose $M_{n}$ and $\alpha_{n}$ as above, let $\beta_{n}:=\left(\mu\left(M_{n}\right)\right)^{-\frac{1}{p^{\prime}}}$, and set

$$
\mathfrak{h}_{n}(\rho):=\beta_{n} \chi_{M_{n}}(\rho) h, \quad \rho \in X .
$$

Then $\mathfrak{h}_{n} \in L^{p^{\prime}}\left(X, \mu, E^{*}\right)$ and $\left\|\mathfrak{h}_{n}\right\|_{L^{p^{\prime}}\left(X, \mu, E^{*}\right)}=\|h\|$. Choose $g \in E$ such that $\langle g, h\rangle=1$ and set

$$
\mathfrak{g}_{n}(\rho):=\alpha_{n} \chi_{M_{n}}(\rho) g, \quad \rho \in X .
$$

Then $\mathfrak{g}_{n} \in L^{p}(X, \mu, E),\left\|\mathfrak{g}_{n}\right\|_{L^{p}(X, \mu, E)}=\|g\|$, and $\mathfrak{f}_{n}:=(\mathfrak{A}-\lambda)^{-1} \mathfrak{g}_{n} \in L^{p}(X, \mu, D)$ because $\mathcal{D}(\mathfrak{A})=L^{p}(X, \mu, D)$ and the closed graph theorem imply that $(\mathfrak{A}-\lambda)^{-1} \in$ $\mathcal{B}\left(\mathfrak{H}, L^{p}(X, \mu, D)\right)$. Thus we would obtain

$$
\begin{aligned}
1 & =|\langle g, h\rangle| \\
& =\beta_{n} \int_{M_{n}}\left|\left\langle\alpha_{n} g, h\right\rangle\right| d \mu(\rho) \\
& =\beta_{n} \int_{M_{n}}\left|\left\langle(A(\rho)-\lambda) \mathfrak{f}_{n}(\rho), h\right\rangle\right| d \mu(\rho) \\
& =\beta_{n} \int_{M_{n}}\left|\left\langle\left(A(\rho)-A\left(\rho_{0}\right)\right) \mathfrak{f}_{n}(\rho), h\right\rangle\right| d \mu(\rho) \\
& =\int_{M_{n}}\left|\left\langle\left(A(\rho)-A\left(\rho_{0}\right)\right) \mathfrak{f}_{n}(\rho), \mathfrak{h}_{n}(\rho)\right\rangle\right| d \mu(\rho) \\
& \leq \int_{M_{n}}\left\|A(\rho)-A\left(\rho_{0}\right)\right\|_{G}\left\|\mathfrak{f}_{n}(\rho)\right\|_{G}\left\|\mathfrak{h}_{n}(\rho)\right\| d \mu(\rho) \\
& \leq \frac{1}{n}\left\|\mathfrak{f}_{n}\right\|_{L^{p}(X, \mu, D)}\left\|\mathfrak{h}_{n}\right\|_{L^{p^{\prime}}\left(X, \mu, E^{*}\right)} \\
& =\frac{1}{n}\left\|\mathfrak{f}_{n}\right\|_{L^{p}(X, \mu, D)}\|h\|_{,}
\end{aligned}
$$

and hence $\left\|\mathfrak{f}_{n}\right\|_{L^{p}(X, \mu, D)}=\left\|(\mathfrak{A}-\lambda)^{-1} \mathfrak{g}_{n}\right\|_{L^{p}(X, \mu, D)} \rightarrow \infty$ as $n \rightarrow \infty$. This contradiction shows that also in this case $\lambda \in \sigma(\mathfrak{A})$.

Conversely, let $\lambda \in \sigma(\mathfrak{A})$. If $\lambda$ is an eigenvalue of $\mathfrak{A}$, then there exists a non-zero $f \in \mathcal{D}(\mathfrak{A})=L^{p}(X, \mu, D)$ such that $\mathfrak{A} f=\lambda f$. Hence, by definition of $\mathfrak{A}, A(\rho) f(\rho)=\lambda f(\rho)$ for almost all $\rho \in X$. Since $f \neq 0, f(\rho) \neq 0$ for all $\rho$ in some set of positive measure. Hence there is $\rho_{0} \in X$ such that $f\left(\rho_{0}\right) \neq 0$ and 
$A\left(\rho_{0}\right) f\left(\rho_{0}\right)=\lambda f\left(\rho_{0}\right)$. This proves

$$
\sigma_{p}(\mathfrak{A}) \subset \bigcup_{\rho \in X} \sigma_{p}(A(\rho)) .
$$

Now assume that $\lambda \in \sigma(\mathfrak{A})$ is not an eigenvalue of $\mathfrak{A}$. Then $\mathfrak{A}-\lambda$ is injective, but not surjective, and we can find an element $\mathfrak{g} \in \mathfrak{H}$ such that $(\mathfrak{A}-\lambda) \mathfrak{f} \neq \mathfrak{g}$ for all $\mathfrak{f} \in L^{p}(X, \mu, D)$. Assume $\lambda \notin \bigcup_{\rho \in Y} \sigma(A(\rho))$. For (almost all) $\rho \in X$ we define

$$
\mathfrak{h}(\rho):=(A(\rho)-\lambda)^{-1} \mathfrak{g}(\rho) .
$$

From assumption (b) and the continuity of the inversion, see [8, Theorem IV.1.16], it follows that the mapping $\rho \mapsto(A(\rho)-\lambda)^{-1}$ from $Y$ into $\mathcal{B}(E, D)$ is continuous. Hence $\mathfrak{h}: X \rightarrow D$ is measurable and

$$
\left(\int_{X}\|\mathfrak{h}(\rho)\|_{G}^{p} d \mu(\rho)\right)^{\frac{1}{p}} \leq \sup _{\rho \in Y}\left\|(A(\rho)-\lambda)^{-1}\right\|_{G}\|\mathfrak{g}\|_{\mathfrak{H}}<\infty
$$

since $Y$ is compact. Thus, by $(3.2), \mathfrak{h} \in L^{p}(X, \mu, D)=\mathcal{D}(\mathfrak{A})$ and $(\mathfrak{A}-\lambda) \mathfrak{h}=\mathfrak{g}$, a contradiction.

Remark 3.2. i) It is a remarkable fact that the spectrum of $\mathfrak{A}$ is independent of the chosen measure $\mu$ as long as $\operatorname{supp} \mu=X$.

ii) Also, the spectrum of $\mathfrak{A}$ is independent of $p$. This is a property which often holds for differential operators in $L^{p}$ spaces.

iii) The assumption that $\operatorname{supp} \mu=X$ is not essential in that one can replace $X$ with supp $\mu$ and $Y$ with the closure of $\operatorname{supp} \mu$ in $Y$. Assumptions (a) and (b) clearly remain true for these smaller sets.

Example. Let $n \in \mathbb{N},-\infty<a<b<\infty$, let $\left.A \in M_{n}(C[a, b])\right)$ be an $n \times n$ matrix the entries of which are continuous functions on $[a, b]$, and consider the Lebesgue measure $\lambda$ on $[a, b]$. Then the family $A:[a, b] \rightarrow M_{n}(\mathbb{C})$ of matrices satisfies conditions (a) and (b), and the multiplication operator $\mathfrak{A}$ acting in the space $L^{p}\left([a, b], \lambda, \mathbb{C}^{n}\right)=L^{p}([a, b])^{n}$ defined in Theorem 2.3 by

$$
(\mathfrak{A} f)(x)=A(x) f(x)
$$

for $f \in L^{p}([a, b])^{n}$ and almost all $x \in[a, b]$ is the usual operator of multiplication by the matrix function $A$. By Theorem 3.1 it follows that (compare [7]),

$$
\sigma(\mathfrak{A})=\bigcup_{x \in[a, b]} \sigma_{p}(A(x))=\bigcup_{x \in[a, b]}\{\lambda \in \mathbb{C}: \operatorname{det}(A(x)-\lambda)=0\} .
$$

In particular, if $n=1$ and $u \in C[a, b]$, we obtain the well known result that the spectrum of the operator $\mathfrak{A}$ of multiplication by the function $u$ is given by

$$
\sigma(\mathfrak{A})=\bigcup_{x \in[a, b]} u(x)=u([a, b]) .
$$


Proposition 3.3. Let assumptions (a) and (b) be satisfied and suppose in addition that for all $\lambda \notin \overline{\bigcup_{\rho \in X} \sigma(A(\rho))}$ there exists a constant $M_{\lambda}>0$ such that

$$
\sup _{\rho \in X}\left\|(A(\rho)-\lambda)^{-1}\right\|_{G} \leq M_{\lambda} .
$$

Then

$$
\sigma(\mathfrak{A})=\overline{\bigcup_{\rho \in X} \sigma(A(\rho))} .
$$

Proof. The inclusion

$$
\sigma(\mathfrak{A}) \supset \overline{\bigcup_{\rho \in X} \sigma(A(\rho))}
$$

follows from Theorem 3.1 since $\sigma(\mathfrak{A})$ is closed. For the point spectrum the converse inclusion (even without the closure) has been proved in (3.1). The proof of the inclusion for the whole spectrum follows if we modify the last paragraph of the proof of Theorem 3.1 using assumption (3.3) in order to show in (3.2) that the function $\mathfrak{h}$ therein belongs to $L^{p}(X, \mu, D)$.

In the next theorem we will see that assumption (3.3) is fulfilled if all operators $A(\rho), \rho \in X$, are self-adjoint. However, the following example shows that, even if conditions (a) and (b) hold, it may happen that

$$
\bigcup_{\rho \in Y} \sigma(A(\rho)) \not \subset \overline{\bigcup_{\rho \in X} \sigma(A(\rho))} \text {. }
$$

Example. Consider the family of operators in $\ell^{2}(\mathbb{Z})$ given by

$$
A(\rho)=A_{0}+\rho A_{1}, \quad \rho \in(0,1],
$$

where $A_{0}$ is a modified left shift operator in $\ell^{2}(\mathbb{Z})$ defined by

$$
A_{0} x_{0}=0, \quad A_{0} x_{n}=x_{n-1}, \quad n \in \mathbb{Z}, n \neq 0,
$$

and the operator $A_{1}$ in $\ell^{2}(\mathbb{Z})$ is given by

$$
A_{1} x_{0}=x_{-1}, \quad A_{1} x_{n}=0, \quad n \in \mathbb{Z}, n \neq 0 .
$$

It is not difficult to show, see [8, Chapter IV, Example 3.8], that

$$
\sigma(A(\rho)) \subset\{\lambda \in \mathbb{C}:|\lambda|=1\}, \quad \rho \in(0,1],
$$

but in the limit $\rho \rightarrow 0$ one has

$$
\sigma\left(A_{0}\right)=\{\lambda \in \mathbb{C}:|\lambda| \leq 1\} .
$$

Theorem 3.4. Let $E$ be a Hilbert space and let assumptions (a) and (b) be satisfied. Assume that $A$ is self-adjoint, i. e., $A(\rho)$ is self-adjoint for all $\rho \in X$. Then

$$
\sigma(\mathfrak{A})=\overline{\bigcup_{\rho \in X} \sigma(A(\rho))}
$$

and $\mathfrak{A}$ is self-adjoint if $p=2$. 
Proof. We first note that we can take $p=2$, see Remark $3.2 \mathrm{i})$. Also, the selfadjointness of each $A(\rho)$ implies that for any $\rho \in X$ and $\lambda \notin \overline{\bigcup_{\rho^{\prime} \in X} \sigma\left(A\left(\rho^{\prime}\right)\right)}$ we have the estimate

$$
\left\|(A(\rho)-\lambda)^{-1}\right\|=(\operatorname{dist}(\lambda, \sigma(A(\rho))))^{-1} \leq\left(\operatorname{dist}\left(\lambda, \bigcup_{\rho^{\prime} \in X} \sigma\left(A\left(\rho^{\prime}\right)\right)\right)\right)^{-1}=: \eta<\infty,
$$

where $\eta$ is independent of $\rho$. Then, with the aid of (2.2), it follows that

$$
\begin{aligned}
m_{G}^{2}\left\|(A(\rho)-\lambda)^{-1} x\right\|_{G}^{2} & \leq\left\|(A(\rho)-\lambda)^{-1} x\right\|_{A(\rho)}^{2} \\
& =\left\|(A(\rho)-\lambda)^{-1} x\right\|^{2}+\left\|A(\rho)(A(\rho)-\lambda)^{-1} x\right\|^{2} \\
& =\left\|(A(\rho)-\lambda)^{-1} x\right\|^{2}+\left\|\left(I+\lambda(A(\rho)-\lambda)^{-1}\right) x\right\|^{2} \\
& \leq\left(2+\left(1+2|\lambda|^{2}\right)\left\|(A(\rho)-\lambda)^{-1}\right\|^{2}\right)\|x\|^{2} \\
& \leq\left(2+\left(1+2|\lambda|^{2}\right) \eta^{2}\right)\|x\|^{2}
\end{aligned}
$$

for $\rho \in X$ and $x \in E$ and hence condition (3.3) of Corollary 3.3 is satisfied, which proves the assertion about the spectrum of $\mathfrak{A}$.

It remains to be shown that $\mathfrak{A}$ is self-adjoint. For $\mathfrak{f}, \mathfrak{g} \in L^{2}(X, \mu, D)$ we have

$$
(\mathfrak{A} \mathfrak{f}, \mathfrak{g})=\int_{X}(A(\rho) \mathfrak{f}(\rho), \mathfrak{g}(\rho)) d \mu(\rho)=\int_{X}(\mathfrak{f}(\rho), A(\rho) \mathfrak{g}(\rho)) d \mu(\rho)=(\mathfrak{f}, \mathfrak{A} \mathfrak{g}),
$$

and hence $\mathfrak{A}$ is symmetric. For all $\rho \in X$ we have $\sigma(A(\rho)) \subset \mathbb{R}$ since $A(\rho)$ is self-adjoint. From what has already been proved it follows that $\sigma(\mathfrak{A}) \subset \mathbb{R}$. Since the operator $\mathfrak{A}$ is symmetric and closed, this implies that $\mathfrak{A}$ is self-adjoint.

\section{The point spectrum of the multiplication operator}

In this section we assume that $E$ is a Hilbert space and that $p=2$.

Theorem 4.1. Let assumptions (a) and (b) be satisfied, where $E$ is a separable Hilbert space, and suppose $p=2$. Then $\lambda \in \sigma_{p}(\mathfrak{A})$ if and only if there exists a measurable subset $M$ of $X$ such that $\mu(M)>0$ and

$$
\lambda \in \sigma_{p}(A(\rho)) \quad \text { for all } \rho \in M .
$$

Proof. If $\lambda \in \sigma_{p}(\mathfrak{A})$, we have already seen in the proof of Theorem 3.1 that there exists a set $E$ of positive measure such that (4.1) holds.

Conversely, let $\lambda \in \mathbb{C}$ be given for which a measurable set $M$ with $\mu(M)>0$ and (4.1) exists. Due to the fact that $\mu$ is a Radon measure, every measurable set of infinite measure contains a measurable subset of positive finite measure. Therefore we may assume $\mu(M)<\infty$. We want to show that $\lambda \in \sigma_{p}(\mathfrak{A})$.

The main part of the proof consists in showing that the orthogonal projection in $D$ onto the null space $N(A(\rho)-\lambda)$ is measurable. To see this, for every $\rho \in X$ we consider the operator $A(\rho)-\lambda$ as a bounded operator from $D$ to $E$ and define its 
adjoint operator $(A(\rho)-\lambda)^{*} \in \mathcal{B}(E, D)$. Due to assumption (b) and the definition of the adjoint operator, for every fixed $f \in D$ and $g \in E$ the scalar-valued function

$$
\rho \mapsto\left(f,(A(\rho)-\lambda)^{*} g\right)_{G}
$$

is a continuous (and thus measurable) function on $X$. Here $(\cdot, \cdot)_{G}$ denotes the scalar product in the Hilbert space $D$ induced by $\|\cdot\|_{G}$.

Now we fix an orthonormal basis $\left\{e_{n}\right\}_{1}^{\infty}$ of $E$. Then $\mathfrak{f}_{n}(\rho):=(A(\rho)-\lambda)^{*} e_{n}$ is a measurable function of $\rho$, and for every fixed $\rho \in X$ the set $\left\{\mathfrak{f}_{n}(\rho): n \in \mathbb{N}\right\}$ is complete in the range $R(A(\rho)-\lambda)^{*} \subset D$ in the sense that the closure of all finite linear combinations of $\mathfrak{f}_{n}(\rho)$ contains this range. Applying the Gram-Schmidt orthogonalization to $\left\{\mathfrak{f}_{n}(\rho)\right\}_{1}^{\infty}$, it is possible to construct an orthonormal basis $\left\{\mathfrak{f}_{n}^{\prime}(\rho)\right\}_{1}^{\infty}$ of $\overline{R(A(\rho)-\lambda)^{*}}$ (orthonormal with respect to $(\cdot, \cdot)_{G}$ ) which depends measurably on $\rho$, see [5, Chapter II, $\S 1$, Lemma 1]. This implies the measurability of the orthogonal projection in $D$ onto $\overline{R(A(\rho)-\lambda)^{*}}$, i.e. the measurability of

$$
\rho \mapsto P^{\prime}(\rho) f:=\sum_{n=1}^{\infty}\left(f, \mathfrak{f}_{n}^{\prime}(\rho)\right)_{G} \mathfrak{f}_{n}^{\prime}(\rho) \in D
$$

for every fixed $f \in D$. Therefore, for the projection $P(\rho):=I-P^{\prime}(\rho), \rho \mapsto P(\rho) f$ is also measurable for all $f \in D$. But $P(\rho)$ is the orthogonal projection in $D$ onto

$$
{\overline{R(A(\rho)-\lambda)^{*}}}^{\perp_{D}}=N(A(\rho)-\lambda) .
$$

As $D$ endowed with the norm $\|\cdot\|_{G}$ is isomorphic to the graph of $G$, which is a closed subspace of the separable Hilbert space $E \times E, D$ is separable, too. We fix an orthonormal basis $\left\{h_{n}\right\}_{1}^{\infty}$ of $D$ and define $\mathfrak{f}(\rho):=P(\rho) h_{N(\rho)}$ for $\rho \in M$ where

$$
N(\rho):=\min \left\{n \in \mathbb{N}: P(\rho) h_{n} \neq 0\right\},
$$

adapting an idea from [1], proof of Lemma 5.7. Note that for every $\rho \in M$ at least one $n \in \mathbb{N}$ exists with $P(\rho) h_{n} \neq 0$ because $N(A(\rho)-\lambda) \neq\{0\}$. For $\rho \in X \backslash M$, we define $\mathfrak{f}(\rho):=0$.

As $\rho \mapsto P(\rho) h_{n}$ is measurable for every $n \in \mathbb{N}$, the same is true for $\rho \mapsto \mathfrak{f}(\rho)$. Moreover, we have

$$
\|\mathfrak{f}\|_{L^{2}(X, \mu, D)} \leq \mu(M)^{\frac{1}{2}} \sup _{\substack{\rho \in M \\ n \in \mathbb{N}}}\left\|P(\rho) h_{n}\right\|_{G} \leq \mu(M)^{\frac{1}{2}}<\infty,
$$

and therefore the function $\mathfrak{f}$ belongs to the domain of $\mathfrak{A}$. By definition of $\mathfrak{f}$, we have $(A(\rho)-\lambda) \mathfrak{f}(\rho)=0$ for all $\rho \in X$ and $\mathfrak{f}(\rho) \neq 0$ for all $\rho \in M$ which shows that $\lambda$ is an eigenvalue of $\mathfrak{A}$.

If one takes a singleton $\{\rho\}$ for $X$, then obviously $\mathfrak{A}$ is isomorphic to $A(\rho)$, and $\sigma_{\text {ess }}(A(\rho)) \neq \sigma(A(\rho))$ implies $\sigma_{\text {ess }}(\mathfrak{A}) \neq \sigma(\mathfrak{A})$. Below we shall see that this latter property cannot happen if $\mu$ is non-atomic, i.e., if for every measurable subset $M$ of $X$ with $\mu(M)>0$ there is a measurable subset $M_{0} \subset M$ such that $0<\mu\left(M_{0}\right)<\mu(M)$. 
Theorem 4.2. Let assumptions (a) and (b) be satisfied, where $E$ is a Hilbert space, and suppose $p=2$. Assume that $\mu$ is non-atomic. Then $\sigma_{\mathrm{ess}}(\mathfrak{A})=\sigma(\mathfrak{A})$.

Proof. First we show that every eigenvalue has an infinite dimensional eigenspace. If $(\mathfrak{A}-\lambda) \mathfrak{f}=0, \mathfrak{f} \in \mathfrak{H}, \mathfrak{f} \neq 0$, then choose $M_{1} \supset M_{2} \supset M_{3} \supset \ldots$, measurable subsets of $X$, such that $\mathfrak{f}(\rho) \neq 0$ for all $\rho \in M_{1}$ and $\mu\left(M_{1}\right)>\mu\left(M_{2}\right)>\mu\left(M_{3}\right)>\ldots$ Then $(\mathfrak{A}-\lambda) \mathfrak{f} \chi_{M_{n}}=0 \cdot \chi_{M_{n}}=0$, i. e., $\left\{\mathfrak{f} \chi_{M_{n}}: n \in \mathbb{N}\right\}$ belongs to the null space of $\mathfrak{A}-\lambda$ and is obviously a set of linearly independent functions. Hence $\lambda$ is an eigenvalue with infinite dimensional eigenspace.

Now assume $\mathfrak{f} \in \mathfrak{H} \backslash\{0\}$ is orthogonal to the range of $\mathfrak{A}-\lambda$. Choosing sets $M_{1}, M_{2}, \ldots$ as above we obtain for all $\mathfrak{g} \in \mathcal{D}(\mathfrak{A})$ that

$$
\left((\mathfrak{A}-\lambda) \mathfrak{g}, \mathfrak{f} \chi_{M_{n}}\right)=\left((\mathfrak{A}-\lambda) \mathfrak{g} \chi_{M_{n}}, \mathfrak{f}\right)=0,
$$

i. e., $\left\{\mathfrak{f} \chi_{M_{n}}: n \in \mathbb{N}\right\}$ is orthogonal to the range of $\mathfrak{A}-\lambda$, and thus the range of $\mathfrak{A}-\lambda$ cannot be a proper subspace of $\mathfrak{H}$ with a finite dimensional complement.

Remark 4.3. The statement of Theorem 4.2 remains true for all $1<p<\infty$, all $\sigma$-finite measures $\mu$ and all Banach spaces $E$ with the Radon-Nikodým property since then

$$
\left(L^{p}(X, \mu, E)\right)^{*}=L^{p^{\prime}}\left(X, \mu, E^{*}\right) .
$$

Note that Hilbert spaces have the Radon-Nikodým property, see [4, Corollary IV.1.4]. In case $\mu$ is a finite measure, (4.2) can be found in [4, Theorem IV.1.1]; this easily extends to $\sigma$-finite measures.

\section{The spectrum of $\mathfrak{A}$ under weakened assumptions}

In the previous sections we assumed that $A$ is defined on $Y$. But the operators $A(\rho)$ are naturally defined only on $X$, and even though continuous dependence on $\rho$ might be a reasonable assumption, requiring that we have a continuos extension to $Y$ could be too restrictive. However, the estimates (2.2) are essential to define $\mathfrak{A}$ and to show that $\mathfrak{A}$ is closed. Hence we shall consider the conditions

(a') $D=\mathcal{D}(A(\rho)), \rho \in X$, is independent of $\rho$ and a dense subspace of $E$,

$\left(\mathrm{b}^{\prime}\right) A: X \rightarrow \mathcal{C}_{D}(E)$ is continuous,

(c') There are positive constants $m_{G}$ and $M_{G}$ such that

$$
m_{G}\|x\|_{G} \leq\|x\|_{A(\rho)} \leq M_{G}\|x\|_{G}, \quad x \in D, \rho \in X .
$$

It is clear from Proposition 2.1 that the assumptions $\left(\mathrm{a}^{\prime}\right),\left(\mathrm{b}^{\prime}\right),\left(\mathrm{c}^{\prime}\right)$ are weaker than the assumptions (a), (b).

It is now easy to see that

Remark 5.1. The statements of Proposition 2.1 and Theorem 2.3 remain true if the assumptions (a) and (b) are replaced by $\left(a^{\prime}\right),\left(b^{\prime}\right)$, and $\left(c^{\prime}\right)$.

Revisiting the proofs of Theorem 3.1 and Proposition 3.3, we obtain 
Corollary 5.2. Let assumptions $\left(\mathrm{a}^{\prime}\right),\left(\mathrm{b}^{\prime}\right)$, and $\left(\mathrm{c}^{\prime}\right)$ be satisfied. Then

$$
\overline{\bigcup_{\rho \in X} \sigma(A(\rho))} \subset \sigma(\mathfrak{A}) \quad \text { and } \quad \sigma_{p}(\mathfrak{A}) \subset \bigcup_{\rho \in X} \sigma_{p}(A(\rho)) \text {. }
$$

If, additionally, for all $\lambda \notin \overline{\bigcup_{\rho \in X} \sigma(A(\rho))}$ there exists a constant $M_{\lambda}>0$ such that

$$
\sup _{\rho \in X}\left\|(A(\rho)-\lambda)^{-1}\right\|_{G} \leq M_{\lambda}
$$

then

$$
\sigma(\mathfrak{A})=\overline{\bigcup_{\rho \in X} \sigma(A(\rho))}
$$

Finally, we note

Remark 5.3. In Theorems 3.4, 4.1 and 4.2 assumptions (a) and (b) can be replaced by $\left(\mathrm{a}^{\prime}\right),\left(\mathrm{b}^{\prime}\right)$, and $\left(\mathrm{c}^{\prime}\right)$.

\section{Examples}

6.1. Let $I=[a, b]$ and $X$ be intervals, $-\infty<a<b<\infty, \mu$ a Radon measure on $X$ with support $X, 1 \leq p<\infty, n \in \mathbb{N}$, and set $\mathfrak{H}=\left(L^{p}(X \times I, \mu)\right)^{n}$ (actually, the measure should be the product measure $\mu \times$ Lebesgue measure, but the notation for Lebesgue measure will always be suppressed). We note that, by Fubini's theorem, $\mathfrak{H}=\left(L^{p}\left(X, \mu, L^{p}(I)\right)\right)^{n}$. Let $Y$ be the standard compactification of $X$ in $\overline{\mathbb{R}}$. Consider a continuous function $B: Y \times I \rightarrow M_{n}(\mathbb{C})$, where $M_{n}(\mathbb{C})$ denotes the set of $n \times n$ matrices with entries in $\mathbb{C}$, and define the operator $\mathfrak{A}$ on $\mathfrak{H}$ by

$$
\begin{aligned}
\mathcal{D}(\mathfrak{A}) & =\left\{f \in\left(L^{p}(X \times I, \mu)\right)^{n}: \partial_{2} f \in\left(L^{p}(X \times I, \mu)\right)^{n}, f(\cdot, a)=f(\cdot, b)\right\}, \\
\mathfrak{A} f & =\partial_{2} f-B f, \quad f \in \mathcal{D}(\mathfrak{A}),
\end{aligned}
$$

where $\partial_{2}$ denotes differentiation with respect to the second variable.

For each $\rho \in Y$ define $A(\rho)$ by

$$
\begin{gathered}
\mathcal{D}(A(\rho))=\left\{g \in\left(W^{1, p}(I)\right)^{n}: g(a)=g(b)\right\}, \\
A(\rho) g=g^{\prime}-B(\rho, \cdot) g, \quad g \in \mathcal{D}(A(\rho)),
\end{gathered}
$$

where $W^{1, p}(I)$ is the usual Sobolev space.

Theorem 6.1. The operator $\mathfrak{A}$ is closed, $\sigma(\mathfrak{A})=\sigma_{\mathrm{ess}}(\mathfrak{A})$, and

$$
\sigma(\mathfrak{A})=\bigcup_{\rho \in Y} \sigma(A(\rho))=\overline{\bigcup_{\rho \in X} \sigma(A(\rho))} .
$$

Proof. Each of the operators $A(\rho)$ is a closed operator since it is a relatively compact perturbation of the system of differential operators with $B$ replaced by zero. From the continuity of $B$ on $Y \times I$ it follows immediately that $A$ depends continuously on $\rho$ as a mapping from $Y$ into $\mathcal{C}_{W^{1, p}(I)}\left(L^{p}(I)\right)$. Observe that $f \in$ $\mathcal{D}(\mathfrak{A})$ if and only if $f, \partial_{2} f \in\left(L^{p}(X \times I, \mu)\right)^{n}$, i. e., $f \in L^{p}\left(X, \rho, W^{1, p}(I)\right)$. Hence 
$\mathfrak{A}$ is the multiplication operator associated with the family $A(\rho)_{\rho \in X}$ and therefore closed by Theorem 2.3. The assertion on the essential spectrum and the left identity above follow from Theorems 3.1, 4.2, and Remark 4.3.

To prove the right identity we first note that neither side of this equation depends on $p$. Therefore it is sufficient to show that $\left(\mathrm{c}^{\prime}\right)$ holds for $p=2$. To this end let $g \in\left(W^{1,2}(I)\right)^{n}$. Then

$$
\begin{aligned}
\|g\|_{\left(W^{1,2}(I)\right)^{n}}^{2} & =\|g\|_{\left(L^{2}(I)\right)^{n}}^{2}+\left\|g^{\prime}\right\|_{\left(L^{2}(I)\right)^{n}}^{2} \\
& \leq\|g\|_{\left(L^{2}(I)\right)^{n}}^{2}+2\left\|g^{\prime}-B(\rho, \cdot) g\right\|_{\left(L^{2}(I)\right)^{n}}^{2}+2\|B(\rho, \cdot) g\|_{\left(L^{2}(I)\right)^{n}}^{2} \\
& \leq\|g\|_{\left(L^{2}(I)\right)^{n}}^{2}+2\|A(\rho) g\|_{\left(L^{2}(I)\right)^{n}}^{2}+2 \sup _{\substack{\rho \in X \\
t \in I}}^{2}\|B(\rho, t)\|^{2}\|g\|_{\left(L^{2}(I)\right)^{n}}^{2} \\
& \leq 2\left(1+\sup _{\substack{\rho \in X \\
t \in I}}\|B(\rho, t)\|^{2}\right)\|g\|_{A(\rho)}^{2},
\end{aligned}
$$

where $\|B(\rho, t)\|$ denotes the matrix operator norm associated with the Euclidean norm on $\mathbb{C}^{n}$. Similarly,

$$
\|g\|_{A(\rho)}^{2} \leq 2\left(1+\sup _{\substack{\rho \in X \\ t \in I}}\|B(\rho, t)\|^{2}\right)\|g\|_{\left(W^{1,2}(I)\right)^{n}}^{2}
$$

6.2. Let $I=[a, b]$ and $X$ be intervals, $-\infty<a<b<\infty, \mu$ a Radon measure on $X$ with support $X, n \in \mathbb{N}$, and set $\mathfrak{H}=L^{2}(X \times I, \mu)$. Consider continuous and bounded functions $a_{j}: X \times I \rightarrow \mathbb{C}, j=0, \ldots, n$, such that $a_{n}$ is never zero and $a_{n}^{-1}$ is bounded, and define the operator $\mathfrak{A}$ on $\mathfrak{H}$ by

$$
\begin{aligned}
\mathcal{D}(\mathfrak{A}) & =\left\{\begin{array}{l}
f \in L^{2}(X \times I, \mu): \partial_{2}^{(j)} f \in L^{2}(X \times I, \mu), f^{(j-1)}(\cdot, a)=f^{(j-1)}(\cdot, b), \\
j
\end{array}=1, \ldots, n\right\}, \\
\mathfrak{A} f & =\sum_{j=0}^{n} a_{j} \partial_{2}^{(j)} f, \quad f \in \mathcal{D}(\mathfrak{A}),
\end{aligned}
$$

where $\partial_{2}$ denotes differentiation with respect to the second variable.

For each $\rho \in X$ define $A(\rho)$ by

$$
\begin{aligned}
\mathcal{D}(A(\rho)) & =\left\{g \in\left(H^{n}(I)\right)^{n}: g^{(j-1)}(a)=g^{(j-1)}(b), j=1, \ldots, n\right\}, \\
A(\rho) g & =\sum_{j=0}^{n} a_{j}(\rho, \cdot) g^{(j)}, \quad g \in \mathcal{D}(A(\rho)) .
\end{aligned}
$$

As above we obtain

Theorem 6.2. The operator $\mathfrak{A}$ is closed, $\sigma(\mathfrak{A})=\sigma_{\mathrm{ess}}(\mathfrak{A})$, and

$$
\sigma(\mathfrak{A})=\overline{\bigcup_{\rho \in X} \sigma(A(\rho))} .
$$

The picture, however, changes if $a_{n}$ is allowed to have zeros. In this case, the domains of the operators $A(\rho)$ are no longer independent of $\rho$. This problem will be consider in a forthcoming paper. 


\section{References}

[1] Azoff, E. A., Spectrum and direct integral, Trans. Amer. Math. Soc. 197 (1974), 211-223.

[2] Binding, P., Volkmer, H., A spectrum determined by eigencurves, Math. Nachr. 202 (1999), 1-15.

[3] Denk, R., Möller, M., Tretter, C., The spectrum of the multiplication operator associated with a family of operators, J. London Math. Soc. 65:2 (2002), 483-492.

[4] Diestel, J., Uhl, J. J., Vector measures, American Math. Soc., Providence, Rhode Island, 1977.

[5] Dixmier, J., Les algèbres d'opérateurs dans l'espace hilbertien (algèbres de von Neumann), Deuxième édition, Gauthier-Villars Éditeur, Paris, 1969.

[6] Dunford, N., Schwartz, J., Linear operators, Part I, Wiley

[7] Hardt, V., Wagenführer, E., Spectral properties of a multiplication operator, Math. Nachr. 178 (1996), 135-156.

[8] Kato, T., Perturbation theory for linear operators, $2^{\text {nd }}$ ed., Springer-Verlag, Berlin, 1980.

[9] Reed, M., Simon, B., Methods of modern mathematical physics. IV. Analysis of operators, Academic Press, New York, 1978.

R. Denk

Fachbereich Mathematik und Statistik

Universität Konstanz

78457 Konstanz

Germany

e-mail: robert.denk@uni-konstanz.de

M. Möller

School of Mathematics

University of the Witwatersrand

2050 WITS

South Africa

e-mail: manfred@maths.wits.ac.za

C. Tretter

FB 3 - Mathematik

Universität Bremen

Bibliothekstr. 1

28359 Bremen

Germany

e-mail: ctretter@math.uni-bremen.de 